\title{
The Use and Value of Mixed Methods Research in Social Work
}

\author{
Josphine Chaumba
}

\begin{abstract}
The complexity of social problems addressed by the social work profession makes mixed methods research an essential tool. This literature review examined common quantitative and qualitative techniques used by social work researchers and what mixed methods research may add to social work research. Surveys and in-depth interviews were the most common quantitative and qualitative data collection methods, respectively. The t-test was the most frequently used quantitative data analysis method. Although thematic analysis was the most common qualitative data analysis method, $12 \%$ of the qualitative data analysis techniques were not specified. Mixed methods research adds three important elements to social work research: voices of participants, comprehensive analyses of phenomena, and enhanced validity of findings. For these reasons, the teaching and use of mixed methods research remain integral to social work.
\end{abstract}

Keywords: Mixed methods research, social work

There is increasing recognition of the importance of combining quantitative and qualitative research methods (hereafter mixed methods research) when conducting social work research and evaluation (Cowger \& Menon, 2001; Grinnell \& Unrau, 2008; Padgett, 1998, 2008; Yegidis \& Weinbach, 2009). At its most basic, mixed methods research refers to research in which investigators use "both qualitative and quantitative approaches or methods in a single study or program of inquiry" (Tashakkori \& Creswell, 2007, p. 4). Nevertheless, potential obstacles to the use of mixed methods research in social work have been reported and these include misunderstanding over components that could be integrated in a single study, and training in either qualitative or quantitative methods but not both (Padgett, 1998).

This article explores the use of mixed methods research in social work through a systematic review of studies that combined quantitative and qualitative methods in published social work journals. The main aims of this literature review were two-fold. First, to understand the common quantitative and qualitative techniques used by social work researchers and second, to explore what mixed methods research may add to social work research. This information may be useful to social workers who are planning to use mixed methods research. Most important, knowledge on how to mix quantitative and qualitative methods is needed so that social workers are equipped to conduct and consume mixed methods research. After this introductory section, an overview of mixed methods research is presented highlighting connections between qualitative, quantitative, and mixed methods research, including when and how mixing occurs. This is followed by the method used to select articles for this review, after which the findings, discussion, and implications of the research effort are articulated.

Josphine Chaumba, Ph.D., is an Assistant Professor in the Department of Social Work at the University of North Carolina at Pembroke in Pembroke, NC.

Copyright (C) 2013 Advances in Social Work Vol. 14 No. 2 (Fall 2013), 307-333 


\section{Review of the Literature}

This literature review provides an overview of key characteristics of qualitative and quantitative methods and their connection to mixed methods in relation to goals, sampling, data collection, and data analysis. Whereas the main goal of quantitative research is to test existing theories and understand connections among particular variables through a deductive research process, primary goals of qualitative research include comprehending multifaceted worlds of study participants and associated subjective meanings and processes using an inductive research process (Padgett, 2008; Rubin \& Babbie, 2008). Clearly there are unique advantages and disadvantages associated with qualitative and quantitative forms of inquiry, which may render each method suited to particular research questions. Hence, "the goal of mixed methods research is to draw on the strengths and minimize the weaknesses of both types of research" (Connelly, 2009, p. 31). As the social work profession advances its understanding of complex social problems such as child abuse, poverty, and substance abuse, mixed methods research will allow exploration of generalizable findings on specific measurable outcomes while capturing the influence of external contexts and subjective processes in a single study (Hopson \& Steiker, 2008).

In view of the above-mentioned goals of quantitative, qualitative, and mixed methods research, sampling, data collection, and analysis methods characteristic of each form of inquiry are reviewed. For instance, the logic underlying quantitative and qualitative sampling varies (Klenke, 2008). The principal reason for sampling in quantitative research is to select individuals that are representative of the population, and this is best achieved through the use of larger sample sizes and random sampling procedures so that the researcher can estimate the representativeness of the sample to facilitate generalizability (Creswell, 2008; Patton, 2002; Rubin \& Babbie, 2008). In contrast, the primary rationale for sampling in qualitative research is to select "information-rich cases for study in-depth" so that the researcher can learn about the issues of central importance to the purpose of the research (Patton, 2002, p. 230). This is best achieved through purposive or theoretical sampling, and the sample size is determined when the point of saturation is reached as marked by redundancy in participants' responses (Klenke, 2008). Consistent with the varying logic between qualitative and quantitative sampling, mixed methods sampling honors the two parallels of representativeness and information-rich cases, and the sample size varies depending on the research strand and questions (Teddlie $\& \mathrm{Yu}, 2007)$.

Even though distinctions between quantitative and qualitative data collection strategies may be blurred because of similar terminology, the form of data that are gathered differs. Whereas qualitative research seeks textual data that capture the context such as words and images, quantitative research collects numbers with less emphasis on context (Creswell \& Plano Clark, 2007; Padgett, 1998). Qualitative and quantitative researchers may use similar terminology when referring to methods of data collection such as surveys, interviews, or observations (Axinn \& Pearce, 2006; Johnson \& Turner, 2003), but differ on issues that are emphasized during the data collection process. For instance, when using observations quantitative researchers may use rating scales or count frequencies whereas qualitative researchers may emphasize processes or interactions in 
the setting. Similarly, quantitative surveys typically consist of structured questionnaires with embedded standardized scales, whereas qualitative surveys use open-ended questions (Weathington, Cunningham, \& Pittenger, 2010). Given the differences in the type of data collected for quantitative versus qualitative research, mixed methods research emphasizes the collection of multiple forms of data, such as both numbers and words or images (Johnson \& Christensen, 2007). In addition, there are specific decisions to be made at this stage, especially for concurrent studies, regarding whether the same types of questions or concepts will be used to collect data for both strands (Creswell \& Plano Clark, 2011).

There are marked differences in the data analysis procedures for quantitative research and qualitative research. Quantitative data analysis seeks to quantify phenomena including identifying statistical relationships among variables, differences between groups, or change over time, whereas qualitative data analysis aims at making sense of the text by searching for themes and patterns in the data (Creswell, 2008; Johnson \& Christensen, 2007). Methods of qualitative data analysis include thematic coding, grounded theory coding, and narrative analysis (Flick, 2009). On the other hand, quantitative data analysis methods can be categorized as descriptive and inferential statistics. Whereas descriptive statistics summarize how variables of interest are distributed in the sample by describing what the data show, inferential statistics are used to make conclusions about the data. Examples of descriptive statistics include frequencies, mean, median, and standard deviation, while statistical tests such as analysis of variance (ANOVA), chi-square, $t$-tests, Pearson's product moment correlation $(r)$, and regression are examples of inferential statistics (Rubin \& Babbie, 2008). Although mixed methods researchers continue to rely on the unique methods of quantitative or qualitative data analysis, they tend to use a variety of data analysis techniques from both forms of inquiry in a single study (Creswell \& Plano Clark, 2011).

Having compared key characteristics of qualitative, quantitative, and mixed methods research, this section delves deeper into the process of doing mixed methods research. Prominent scholars in this form of inquiry have provided guidance on how to combine qualitative and quantitative methods (Cowger \& Menon, 2001; Creswell \& Plano Clark, 2007, 2011; Greene, 2007; Padgett, 1998, 2008). Foremost are the key decisions that researchers need to resolve prior to conducting a mixed methods study, and these are: (1) whether the quantitative and qualitative methods will be implemented at the same time (concurrent), in two distinct phases (sequential), or in three or more phases that combine concurrent and sequential elements (multiphase), (2) the relative weight of the two approaches, that is, whether there will be more emphasis on one method over the other or both methods will have equal weighting, and (3) when and how the quantitative and qualitative methods will be mixed (Creswell \& Plano Clark, 2007, 2011). Determination of how and when mixing occurs is discussed in the remainder of this section.

Lodico, Spaulding, and Voegtle (2006) caution that "just adding a couple of openended questions to a quantitative measure does not constitute a true mixed-methods study" (p. 282). In order to clarify how and when mixing occurs, Creswell and Plano Clark (2011) envisage four possible points. It is worth noting that a study may have 
primary and secondary points of mixing, and examples of primary points of mixing from the reviewed studies are included.

The first possible point of integration is at the design level, where the overall plan of the research involves embedding one approach within a design based on the other type. For instance, a qualitative technique can be embedded within an experimental design, as illustrated by Sanders and Roach (2007). In their evaluation of a family support services intervention, Sanders and Roach used a quantitative pre-test post-test non-equivalent groups design with embedded qualitative methods.

The second point of integration is during data collection, where results from one strand are used to shape the research questions, sampling, or data collection instruments for the other strand. For example, a researcher may start off with in-depth interviews and use the findings from this qualitative study to construct a survey instrument for use in the quantitative part, or conduct a survey and use the results to identify cases for follow-up, in-depth interviews. Varas-Díaz and Marzán-Rodríguez (2007) developed an instrument, Emotions Associated with AIDS Inventory, for use in the quantitative part of the study, drawing from the findings from in-depth interviews that had explored practitioner emotions associated with interacting with people living with HIV/AIDS. The third point of integration is during data analysis. At this stage results from a qualitative study can be transformed to numerical data and analyzed using quantitative methods, as demonstrated by Redman (2008) who used categories from qualitative findings as the dependent variables for quantitative data analysis.

The fourth point of integration is during interpretation, where the researcher merges the qualitative and quantitative strands by comparing or contrasting findings from the two data sets. While this is the only point of integration for researchers who use procedures with separate qualitative and quantitative strands, synthesizing what was learned from mixing the two methods in the discussion section is anticipated in mixed methods studies (Creswell \& Plano Clark, 2011). For instance, McAuley, McCurry, Knapp, Beecham and Sleed (2006) discuss how the data from the two methods converged. On the other end, Nicotera (2008) discusses how mixed methods research aided in understanding differences between neighborhoods. It is important to note that merging findings at the interpretation stage can be challenging, as Padgett (2009) stated, "Perhaps the most daunting challenge is integrating findings from the two 'sides', especially, when the findings conflict" (p. 104).

Given the potential challenges associated with mixed methods research, what does mixed methods research add to social inquiry that qualitative or quantitative methods alone may not achieve? To answer this question, the value of mixed methods research is considered drawing from literature on the purposes or rationales that drive the use of mixed methods inquiry and mixed methods research designs. A useful framework for classifying the purposes of mixed methods research was devised by Greene, Caracelli, and Graham (1989) and also reported by Greene (2007). Table 1 summarizes the five main purposes of mixed methods research, highlighting their descriptions, goals, and benefits in a table format for comparison and clarification. 
Table 1. Purposes of Mixed Methods Research Adapted from Greene (2007) and Desimone (2009)

\begin{tabular}{|c|c|c|c|}
\hline Purpose & Description & Goal & Benefit \\
\hline Complementarity & $\begin{array}{l}\text { Different methods are } \\
\text { used for different facets } \\
\text { of the same phenomena }\end{array}$ & $\begin{array}{l}\text { Enrichment, } \\
\text { elaboration, or } \\
\text { clarification of } \\
\text { results }\end{array}$ & $\begin{array}{l}\text { Increases the depth and } \\
\text { confidence in } \\
\text { interpretation as results } \\
\text { from one method clarify } \\
\text { or illustrate results from } \\
\text { the other method }\end{array}$ \\
\hline Development & $\begin{array}{l}\text { Involves the sequential } \\
\text { use of different methods } \\
\text { in the development of } \\
\text { the study for sampling or } \\
\text { instrumentation purposes }\end{array}$ & $\begin{array}{l}\text { Use the results of } \\
\text { one method to } \\
\text { inform the other } \\
\text { method }\end{array}$ & $\begin{array}{l}\text { Takes advantage of } \\
\text { inherent method strengths } \\
\text { for better understanding; } \\
\text { e.g., results from a } \\
\text { questionnaire can be used } \\
\text { to identify issues for in- } \\
\text { depth study }\end{array}$ \\
\hline Expansion & $\begin{array}{l}\text { Different methods are } \\
\text { used for different } \\
\text { phenomena or questions }\end{array}$ & $\begin{array}{l}\text { Expand the scope } \\
\text { or breadth of a } \\
\text { study }\end{array}$ & $\begin{array}{l}\text { Enables study to answer } \\
\text { more questions of interest }\end{array}$ \\
\hline Initiation & $\begin{array}{l}\text { Different methods are } \\
\text { used for different facets } \\
\text { of the same phenomena } \\
\text { with the goal of } \\
\text { identifying contradiction }\end{array}$ & $\begin{array}{l}\text { Non-convergence } \\
\text { of results }\end{array}$ & $\begin{array}{l}\text { May lead to new questions } \\
\text { or rephrasing of the } \\
\text { problem or phenomena } \\
\text { under study }\end{array}$ \\
\hline Triangulation & $\begin{array}{l}\text { Use of mixed methods to } \\
\text { answer the same } \\
\text { question }\end{array}$ & $\begin{array}{l}\text { Correspondence } \\
\text { of results across } \\
\text { different methods }\end{array}$ & $\begin{array}{l}\text { Enhanced validity of } \\
\text { results as the combined } \\
\text { methods offset biases of } \\
\text { either a quantitative or } \\
\text { qualitative only study }\end{array}$ \\
\hline
\end{tabular}

Researchers may have multiple purposes for using mixed methods in a single study, such that there can be primary and secondary reasons for the choice of mixed methods (Bryman, 2006; Greene, 2007). A review of 232 mixed methods studies by Bryman (2006) using Greene and colleagues' framework established that complementarity and expansion and were the most frequently cited reasons accounting for $29 \%$ and $25 \%$ of the studies, respectively. It is important to note that following this review of 232 articles, Bryman developed an expanded classification of possible purposes of mixed methods research that can be a useful resource when planning a mixed methods study. Stating at least one reason for adopting mixed methods research is critical when planning and reporting a mixed methods research study (Creswell \& Plano Clark, 2011), because, when the rationale is explicated, readers are presented with an opportunity to assess the value of combining quantitative and qualitative methods. 
Apart from relying on the frameworks of Greene and colleagues (1989) or Bryman (2006) to identify the rationale for a mixed methods study, articulating the adopted mixed methods research design may point to the underlying primary purpose of a mixed methods study (Creswell \& Plano Clark, 2011). For instance, Creswell and Plano Clark (2007) distinguished four main types of mixed methods research designs with associated rationales, and these are:

1. Triangulation, Concurrent, or Parallel Design which entails separate quantitative and qualitative data collection and analysis within the same timeframe, and merging of data during interpretation for various reasons that may include validating findings from one method, gaining a complete understanding of phenomenon under study, or confirming findings.

2. Embedded Design, a concurrent design where a qualitative part is embedded in a quantitative study, or vice versa, so that the findings of one part (e.g. qualitative) are used to support or explain findings from the other method. This can take the form of an experiment with embedded interviews or observations to understand the process or participant experiences. This design can be useful in research that seeks to develop or gain a complete understanding of interventions.

3. Exploratory Sequential Design, a sequential design in which a qualitative study conducted in the first phase informs a quantitative study conducted in the second phase. When using this design, qualitative findings can be used to guide the development of a quantitative instrument or theory development when hypotheses from qualitative findings are validated or tested using quantitative methods.

4. Explanatory Sequential Design, a sequential design in which a quantitative study conducted in the first phase informs a qualitative study conducted in the second phase. With this design, the results of the qualitative design can be used to explain quantitative findings, or quantitative findings can be used to guide sample selection for the qualitative part (Creswell \& Plano Clark, 2007, 2011)

In sum, if researchers clearly define their mixed methods design and its associated primary purpose, the potential value of combining qualitative and quantitative methods is expressed in addition to enhancing the study's rigor and quality (Creswell \& Plano Clark, 2011). Having highlighted the key characteristics of qualitative and quantitative research, the question of when and how mixing occurs, and the purposes of mixed methods research, the method and findings of the systematic review are presented next.

\section{Method}

A systematic literature search in Social Work Abstracts, PsycINFO, Academic Search Complete, MEDLINE, Family \& Society Studies Worldwide, Sociological Collection, CINAHL, Family Studies Abstracts, and PsycARTICLES was conducted to identify research articles that had used mixed methods research. The literature search was conducted in August 2010 using the EBSCOhost's advanced search option with "mixed methods" and "social work" as the search terms. The search yielded 585 articles published between 1995 and 2010. After the removal of duplicates and limiting the 
search to peer reviewed and full text articles, the sample was reduced to 119. Abstracts were then reviewed to identify research studies that had combined qualitative and quantitative methods. Conceptual articles and literature reviews were excluded during this stage of the review leaving 68 full text articles that were further assessed for eligibility. During this stage, 21 articles were excluded. Figure 1 shows a PRISMA Statement flow diagram of the article selection process according to the guidelines by Moher, Liberati, Tetzlaff, Altman, and The PRISMA Group (2009).

Figure 1: The Prisma Statement Illustrating the Flow of Information Through the Different Phases of the Systematic Review.

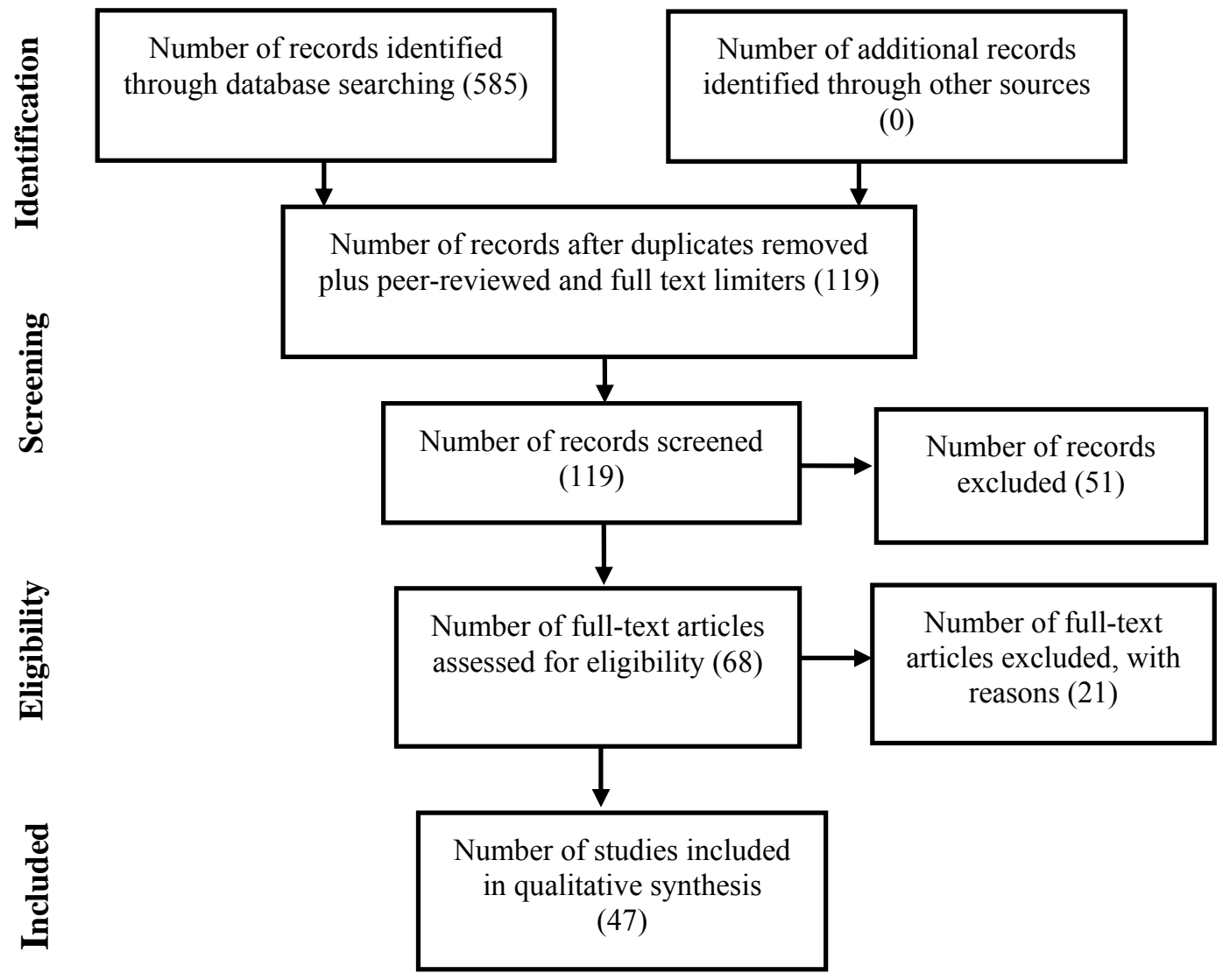

Articles were included in the synthesis if the study procedures involved the use of both quantitative and qualitative techniques and demonstrated at least one point of integration either at the design level, during data collection or analysis, or interpretation in accordance with the classification by Creswell and Plano Clark (2011). In addition, the articles were reviewed for two other key mixed methods research features. First, the purpose of mixed methods research was evaluated using the framework by Greene and colleagues (1989) that identifies complementarity, triangulation, expansion, initiation, and development as the main purposes of mixed methods research. Second, the type of 
mixed methods research was determined using the classification by Creswell and Plano Clark (2007) emphasizing four research designs, namely, triangulation/concurrent/ parallel design, embedded design, exploratory sequential design, and explanatory sequential design. Data were extracted using an adapted coding scheme informed by O'Cathain, Murphy, and Nicholl (2007). The coding form included the following categories, focus of study, purpose or rationale for mixing methods, type of mixed methods design, focus of the quantitative and qualitative parts, point where mixing occurred, qualitative methods of data collection and analysis, and quantitative methods of data collection. Table 2 summarizes the 47 articles that were synthesized in this literature review in line with the categories in the coding form.

After completing the literature review table, frequency counts were performed to establish the types and number of times specific qualitative and quantitative methods were used in the reviewed articles. In addition, drawing from the stated foci of the qualitative and quantitative parts, the researcher inferred elements that mixed methods research may add to social work. The study's main findings are presented next.

\section{Results}

To understand where mixing occurred in the reviewed studies, analyses of the extracted data showed that the most frequently used point of integration was the interpretation stage, accounting for $62 \%(\mathrm{n}=33)$ of all the 53 points of integration represented in the articles. Integration at the design level accounted for $24 \%(\mathrm{n}=13)$ and data collection and data analysis stages accounted for $13 \%(n=7)$ each. The other main findings of this review are organized according to: 1) common methods of qualitative and quantitative data collection, 2) common methods of qualitative and quantitative data analysis, and 3) key areas addressed by the qualitative and quantitative strands. 
Table 2. Published Social Work Journal Articles used in the Literature Review

\begin{tabular}{|c|c|c|c|c|c|c|c|c|}
\hline Author & Focus of Study & $\begin{array}{l}\text { Primary } \\
\text { Purpose of } \\
\text { Mixed Methods } \\
\text { Research }\end{array}$ & $\begin{array}{l}\text { Mixed } \\
\text { Methods } \\
\text { Research } \\
\text { Design }\end{array}$ & $\begin{array}{l}\text { Focus of } \\
\text { Qualitative } \\
\text { Part }\end{array}$ & $\begin{array}{c}\text { Focus of } \\
\text { Quantitative } \\
\text { Part }\end{array}$ & $\begin{array}{c}\text { Where } \\
\text { Mixing } \\
\text { Occurred }\end{array}$ & $\begin{array}{l}\text { Qualitative } \\
\text { Data } \\
\text { Collection \& } \\
\text { Analysis } \\
\text { Methods } \\
\end{array}$ & $\begin{array}{l}\text { Quantitative Data } \\
\text { Collection \& } \\
\text { Analysis Methods }\end{array}$ \\
\hline $\begin{array}{l}\text { Abel \& } \\
\text { Campbell } \\
\text { (2009) }\end{array}$ & $\begin{array}{l}\text { Assessment of } \\
\text { teaching } \\
\text { approaches }\end{array}$ & Triangulation & Triangulation & $\begin{array}{l}\text { Students' } \\
\text { perceptions }\end{array}$ & $\begin{array}{l}\text { - Students } \\
\text { Perceptions } \\
\text {-Differences } \\
\text { in outcomes }\end{array}$ & Interpretation & $\begin{array}{l}\text { Focus Groups } \\
\text { Open \& Axial } \\
\text { Coding }\end{array}$ & $\begin{array}{l}\text { Survey } \\
\text { Descriptive and } \\
\text { Inferential statistics } \\
\text {-Chi Square Test }\end{array}$ \\
\hline $\begin{array}{l}\text { Antle \& } \\
\text { Collins (2009) }\end{array}$ & $\begin{array}{l}\text { Evaluation of a } \\
\text { breast cancer } \\
\text { support group }\end{array}$ & Complementarity & Embedded & $\begin{array}{l}\text { Respondents' } \\
\text { perspectives } \\
\text { and } \\
\text { experiences }\end{array}$ & $\begin{array}{l}\text {-Measure } \\
\text { key variables } \\
\cdot \text { Examine } \\
\text { variable } \\
\text { relationships }\end{array}$ & Design level & \multicolumn{2}{|c|}{$\begin{array}{l}\text { Survey questionnaire with qualitative } \\
\text { questions } \\
\text { Thematic analysis } \\
\text { Descriptive and inferential statistics- } \\
\text { ANOVA and Pearson's } r\end{array}$} \\
\hline $\begin{array}{l}\text { Ayón \& Lee } \\
\text { (2009) }\end{array}$ & $\begin{array}{l}\text { Evaluation of a } \\
\text { Neighborhood } \\
\text { Leadership } \\
\text { Program }\end{array}$ & Expansion & Explanatory & $\begin{array}{l}\text { Participants' } \\
\text { experiences } \\
\text { on use of } \\
\text { skills }\end{array}$ & $\begin{array}{l}\text { Measure } \\
\text { Leadership } \\
\text { Skills and } \\
\text { Knowledge }\end{array}$ & Design level & $\begin{array}{l}\text { In-depth } \\
\text { interviews } \\
\text { Open-coding }\end{array}$ & $\begin{array}{l}\text { Survey } \\
\text { Descriptive and } \\
\text { inferential statistics } \\
\text { - T-test }\end{array}$ \\
\hline $\begin{array}{l}\text { Beecher } \\
(2009 a)\end{array}$ & $\begin{array}{l}\text { Influence of the } \\
\text { medical model on } \\
\text { treatment of } \\
\text { individuals with } \\
\text { schizophrenia }\end{array}$ & Complementarity & Triangulation & $\begin{array}{l}\text { Practitioner } \\
\text { views }\end{array}$ & $\begin{array}{l}\text { Measure key } \\
\text { variables }\end{array}$ & Interpretation & $\begin{array}{l}\text { Web-based } \\
\text { survey } \\
\text { Thematic } \\
\text { analysis }\end{array}$ & $\begin{array}{l}\text { Secondary data } \\
\\
\text { Descriptive and } \\
\text { inferential statistics } \\
\text { - ANOVA } \\
\text { - Kruskal Wallis } \\
\end{array}$ \\
\hline $\begin{array}{l}\text { Beecher } \\
(2009 b)\end{array}$ & $\begin{array}{l}\text { Practitioner views } \\
\text { toward families } \\
\text { and barriers to } \\
\text { collaboration }\end{array}$ & Complementarity & Triangulation & $\begin{array}{l}\text { Practitioner } \\
\text { experiences \& } \\
\text { views }\end{array}$ & $\begin{array}{l}\text { Examine } \\
\text { variable } \\
\text { relationships }\end{array}$ & Interpretation & $\begin{array}{l}\text { Web- based } \\
\text { survey } \\
\text { Thematic } \\
\text { analysis }\end{array}$ & $\begin{array}{l}\text { Survey } \\
\text { Descriptive and } \\
\text { Inferential statistics } \\
\text { • ANOVA } \quad \cdot t \text { - test }\end{array}$ \\
\hline
\end{tabular}




\begin{tabular}{|c|c|c|c|c|c|c|c|c|}
\hline $\begin{array}{l}\text { Bellamy et al. } \\
\text { (2006) }\end{array}$ & $\begin{array}{l}\text { Effects of group } \\
\text { leadership on } \\
\text { group functioning }\end{array}$ & Triangulation & Triangulation & $\begin{array}{l}\text { Group } \\
\text { activities and } \\
\text { interactions }\end{array}$ & $\begin{array}{l}\text { Exploration } \\
\text { of } \\
\text { differences }\end{array}$ & Data Analysis & $\begin{array}{l}\text { Observations } \\
\text { Coding }\end{array}$ & $\begin{array}{l}\text { Transformed } \\
\text { qualitative codes } \\
\text { Descriptive and } \\
\text { Inferential statistics } \\
\cdot t \text { - test } \bullet \text { Pearson's } r \\
\cdot \text { Chi-Square }\end{array}$ \\
\hline $\begin{array}{l}\text { Berger, Otto- } \\
\text { Salaj, Stoffel, } \\
\text { Hernandez- } \\
\text { Meier, \& } \\
\text { Gromoske } \\
(2009)\end{array}$ & $\begin{array}{l}\text { Barriers and } \\
\text { facilitators of } \\
\text { transferring } \\
\text { motivational } \\
\text { interviewing into } \\
\text { practice }\end{array}$ & Expansion & Embedded & $\begin{array}{l}\text { Perceived } \\
\text { barriers \& } \\
\text { facilitators } \\
\text { - Needs and } \\
\text { expectations }\end{array}$ & $\begin{array}{l}\text { Measure key } \\
\text { variables }\end{array}$ & Design level & $\begin{array}{l}\text { Focus groups } \\
\text { Grounded } \\
\text { theory }\end{array}$ & $\begin{array}{l}\text { Survey } \\
\text { Descriptive and } \\
\text { Inferential statistics } \\
\text { - Pearson's } r\end{array}$ \\
\hline Boateng (2009) & $\begin{array}{l}\text { Social capital \& } \\
\text { Liberian refugee } \\
\text { women's well- } \\
\text { being }\end{array}$ & Triangulation & Triangulation & $\begin{array}{l}\text { Participants' } \\
\text { experiences \& } \\
\text { shared issues }\end{array}$ & $\begin{array}{l}\text { Measure } \\
\text { dimensions } \\
\text { of social } \\
\text { capital }\end{array}$ & Interpretation & $\begin{array}{l}\text {-In-depth } \\
\text { interviews } \\
\text {-Focus groups } \\
\text {-Photographs } \\
\text { Constant } \\
\text { comparative } \\
\text { analysis }\end{array}$ & $\begin{array}{l}\text { Survey } \\
\text { Descriptive statistics }\end{array}$ \\
\hline $\begin{array}{l}\text { Bryan, } \\
\text { Flaherty, \& } \\
\text { Saunders } \\
(2010)\end{array}$ & $\begin{array}{l}\text { Evaluation of } \\
\text { Adoption Support } \\
\text { for Kentucky } \\
\text { Program }\end{array}$ & Development & Exploratory & $\begin{array}{l}\text { Gather } \\
\text { information to } \\
\text { guide the } \\
\text { development } \\
\text { of a } \\
\text { quantitative } \\
\text { survey }\end{array}$ & $\begin{array}{l}\text { - Examine } \\
\text { survey } \\
\text { instrument's } \\
\text { measurement } \\
\text { structure } \\
\text { - Describe } \\
\text { program } \\
\text { participants }\end{array}$ & $\begin{array}{l}\text { Data } \\
\text { collection }\end{array}$ & $\begin{array}{l}\text { Focus groups } \\
\text { Open coding }\end{array}$ & $\begin{array}{l}\text { Survey } \\
\text { Descriptive and } \\
\text { Inferential statistics } \\
\text { - Cronbach's alpha } \\
\text { - ANOVA }\end{array}$ \\
\hline Butler (2006) & $\begin{array}{l}\text { Evaluation of the } \\
\text { Senior Companion } \\
\text { Program }\end{array}$ & Triangulation & Triangulation & $\begin{array}{l}\text { Participants' } \\
\text { experiences }\end{array}$ & $\begin{array}{l}\text { Measure } \\
\text { key } \\
\text { constructs }\end{array}$ & Interpretation & \multicolumn{2}{|c|}{$\begin{array}{l}\text { Open-ended questions on questionnaire } \\
\text { Open coding } \\
\text { Descriptive and Inferential statistics } \\
\cdot \text { Pearson's } r \quad \cdot t \text {-tests } \bullet \text { chi-square }\end{array}$} \\
\hline
\end{tabular}




\begin{tabular}{|c|c|c|c|c|c|c|c|c|}
\hline $\begin{array}{l}\text { Campbell } \\
(2008)\end{array}$ & $\begin{array}{l}\text { Stakeholder } \\
\text { experiences with } \\
\text { compulsory } \\
\text { admission to } \\
\text { psychiatric } \\
\text { hospitals, and the } \\
\text { use of the Mental } \\
\text { Health Review } \\
\text { Tribunal (MHRT) } \\
\text { in Northern } \\
\text { Ireland }\end{array}$ & Triangulation & Triangulation & $\begin{array}{l}\text { Views of a } \\
\text { range of } \\
\text { stakeholders } \\
\text { e.g. clients and } \\
\text { key informants }\end{array}$ & $\begin{array}{l}\text { Describe } \\
\text { experiences, } \\
\text { knowledge } \\
\text { and views on } \\
\text { the } \\
\text { adequacy of } \\
\text { mental health } \\
\text { law and } \\
\text { policy, and the } \\
\text { Tribunal } \\
\text { system }\end{array}$ & Interpretation & $\begin{array}{l}\text { focus } \\
\text { groups } \\
\cdot \text { key } \\
\text { informant } \\
\text { interviews } \\
\cdot \text { document } \\
\text { analysis } \\
\\
\text { Thematic } \\
\text { Analysis }\end{array}$ & $\begin{array}{l}\text { Survey } \\
\text { Descriptive } \\
\text { Statistics }\end{array}$ \\
\hline $\begin{array}{l}\text { Carpenter, } \\
\text { Barnes, } \\
\text { Dickinson, \& } \\
\text { Wooff }(2006)\end{array}$ & $\begin{array}{l}\text { Evaluation of a } \\
\text { postgraduate } \\
\text { program }\end{array}$ & Expansion & Embedded & $\begin{array}{l}\text { Students' } \\
\text { experiences and } \\
\text { process of } \\
\text { implementation }\end{array}$ & $\begin{array}{l}\text { •Measure key } \\
\text { outcomes } \\
\text { •Assess } \\
\text { changes in } \\
\text { students' } \\
\text { perceptions of } \\
\text { their } \\
\text { knowledge } \\
\text { and skills } \\
\end{array}$ & Design level & $\begin{array}{l}\text { Participant } \\
\text { observation } \\
\text { Focus groups } \\
\text { Individual } \\
\text { interviews } \\
\text { Thematic } \\
\text { analysis }\end{array}$ & $\begin{array}{l}\text { Survey } \\
\text { Descriptive and } \\
\text { Inferential statistics } \\
\text {-ANOVA/ANCOV } \\
\text { A } \\
\text { •t-tests } \\
\text { •Cronbach's alpha }\end{array}$ \\
\hline $\begin{array}{l}\text { Chan, Chi, } \\
\text { Ching, \& Lam } \\
\text { (2010) }\end{array}$ & $\begin{array}{l}\text { Student } \\
\text { perceptions of } \\
\text { learning }\end{array}$ & Complementarity & Embedded & $\begin{array}{l}\text { Students' } \\
\text { transactions }\end{array}$ & $\begin{array}{l}\text { Student's } \\
\text { ratings of the } \\
\text { approach used } \\
\text { to facilitate } \\
\text { learning }\end{array}$ & $\begin{array}{l}\text { Design Level } \\
\text { Interpretation }\end{array}$ & $\begin{array}{l}\text {-Videotape } \\
\text { recordings } \\
\text { - Telephone } \\
\text { interviews } \\
\text { Thematic } \\
\text { analysis }\end{array}$ & $\begin{array}{l}\text { Surveys } \\
\text { Descriptive statistics }\end{array}$ \\
\hline $\begin{array}{l}\text { Chan, Mok, } \\
\text { Po-ying, \& } \\
\text { Man-chun } \\
(2009)\end{array}$ & $\begin{array}{l}\text { Evaluation of } \\
\text { a teaching method }\end{array}$ & Complementarity & Embedded & $\begin{array}{l}\text { Students' } \\
\text { transactions }\end{array}$ & $\begin{array}{l}\text { Measure } \\
\text { effectiveness } \\
\text { of approach }\end{array}$ & Design level & $\begin{array}{l}\text {-Videotape } \\
\text { recordings } \\
\text { Telephone } \\
\text { interviews } \\
\text { Content } \\
\text { analysis }\end{array}$ & $\begin{array}{l}\text { Surveys } \\
\text { Descriptive statistics }\end{array}$ \\
\hline Cheung (2008) & $\begin{array}{l}\text { Resilience among } \\
\text { older immigrant } \\
\text { couples }\end{array}$ & Triangulation & Triangulation & $\begin{array}{l}\text { Respondents' } \\
\text { lived } \\
\text { experiences }\end{array}$ & $\begin{array}{l}\text { Measure key } \\
\text { variables }\end{array}$ & Interpretation & $\begin{array}{l}\text { In-depth } \\
\text { Interviews } \\
\text { Narrative } \\
\text { Analysis }\end{array}$ & $\begin{array}{l}\text { Survey } \\
\text { Descriptive statistics }\end{array}$ \\
\hline
\end{tabular}




\begin{tabular}{|c|c|c|c|c|c|c|c|c|}
\hline Daftary (2009) & $\begin{array}{l}\text { Factors that shape } \\
\text { elected } \\
\text { leaders' decision } \\
\text { making }\end{array}$ & Complementarity & Triangulation & Explore process & $\begin{array}{l}\text { Examine } \\
\text { variable } \\
\text { relationships }\end{array}$ & Interpretation & $\begin{array}{l}\text { Ethnography } \\
\text { No specified } \\
\text { method of } \\
\text { data analysis }\end{array}$ & $\begin{array}{l}\text { Survey } \\
\text { Descriptive and } \\
\text { Inferential statistics } \\
\text {-Structural equation } \\
\text { modeling }\end{array}$ \\
\hline $\begin{array}{l}\text { Fernandez } \\
(2008)\end{array}$ & $\begin{array}{l}\text { Outcomes of } \\
\text { children in foster } \\
\text { care }\end{array}$ & Complementarity & Embedded & $\begin{array}{l}\text { Process of } \\
\text { interactions }\end{array}$ & $\begin{array}{l}\text { Evaluate } \\
\text { outcomes }\end{array}$ & Design level & $\begin{array}{l}\begin{array}{l}\text { In-depth } \\
\text { interviews }\end{array} \\
\text { No specified } \\
\text { method of } \\
\text { data analysis }\end{array}$ & $\begin{array}{l}\text { Survey } \\
\text { Descriptive and } \\
\text { Inferential statistics } \\
\text { - Chi-Square } \\
\text { - T-test }\end{array}$ \\
\hline $\begin{array}{l}\text { Freedman } \\
(2009)\end{array}$ & $\begin{array}{l}\text { Examination of } \\
\text { local food } \\
\text { environments }\end{array}$ & Triangulation & Triangulation & $\begin{array}{l}\text { Participants' } \\
\text { perceptions }\end{array}$ & $\begin{array}{l}\text { Identify the } \\
\text { types of food } \\
\text { stores and } \\
\text { food items }\end{array}$ & Interpretation & $\begin{array}{l}\text { In-depth } \\
\text { Interviews } \\
\text { Thematic } \\
\text { analysis }\end{array}$ & $\begin{array}{l}\text { Food audit-Survey } \\
\text { Descriptive statistics }\end{array}$ \\
\hline $\begin{array}{l}\text { Gallagher, } \\
\text { Malone, \& } \\
\text { Ladner (2009) }\end{array}$ & $\begin{array}{l}\text { Teamwork among } \\
\text { school } \\
\text { psychologists, } \\
\text { counselors, and } \\
\text { social workers }\end{array}$ & Triangulation & Triangulation & $\begin{array}{l}\text { Perceptions of } \\
\text { the team } \\
\text { process }\end{array}$ & $\begin{array}{l}\text { Measure } \\
\text { attitudes and } \\
\text { perceptions } \\
\text { about } \\
\text { teamwork }\end{array}$ & Interpretation & \multicolumn{2}{|c|}{$\begin{array}{l}\text { Open-ended questions on survey } \\
\text { Content analysis } \\
\text { Survey } \\
\text { Descriptive and Inferential statistics } \\
\text { - Pearson's } r\end{array}$} \\
\hline Gioia (2006) & $\begin{array}{l}\text { Work delay in } \\
\text { young adults with } \\
\text { schizophrenia }\end{array}$ & Triangulation & Triangulation & $\begin{array}{l}\text { Participants' } \\
\text { experiences and } \\
\text { meanings of } \\
\text { work }\end{array}$ & $\begin{array}{l}\text { Pre and post } \\
\text { illness history }\end{array}$ & Interpretation & \multicolumn{2}{|c|}{$\begin{array}{l}\text { Semi-structured face to face } \\
\text { interview } \\
\text { Descriptive statistics } \\
\text { (In-depth interview) Thematic } \\
\text { analysis }\end{array}$} \\
\hline $\begin{array}{l}\text { Gioia \& } \\
\text { Brekke (2003) }\end{array}$ & $\begin{array}{l}\text { Knowledge and } \\
\text { use of ADA } \\
\text { provisions among } \\
\text { people with } \\
\text { schizophrenia }\end{array}$ & Triangulation & Triangulation & $\begin{array}{l}\text { Participants' } \\
\text { experiences and } \\
\text { meanings of } \\
\text { work }\end{array}$ & $\begin{array}{l}\text { Pre and post } \\
\text { illness history }\end{array}$ & Interpretation & \multicolumn{2}{|c|}{$\begin{array}{l}\text { Semi-structured face to face } \\
\text { interview } \\
\text { Descriptive statistics } \\
\text { Thematic analysis }\end{array}$} \\
\hline $\begin{array}{l}\text { Hernandez et } \\
\text { al. (2009) }\end{array}$ & $\begin{array}{l}\text { Provision of } \\
\text { workplace } \\
\text { accommodations }\end{array}$ & Complementarity & Triangulation & $\begin{array}{l}\text { Employer } \\
\text { perceptions on } \\
\text { providing } \\
\text { workplace } \\
\text { accommoda- } \\
\text { tions }\end{array}$ & $\begin{array}{l}\text { Data on } \\
\text { accommoda- } \\
\text { tions }\end{array}$ & Interpretation & $\begin{array}{l}\text { Focus group } \\
\text { Content } \\
\text { analysis }\end{array}$ & $\begin{array}{l}\text { Survey } \\
\text { Descriptive statistics }\end{array}$ \\
\hline
\end{tabular}




\begin{tabular}{|c|c|c|c|c|c|c|c|c|}
\hline $\begin{array}{l}\text { Hodge \& } \\
\text { Boddie (2007) }\end{array}$ & $\begin{array}{l}\text { Personal spiritual } \\
\text { characteristics and } \\
\text { understanding of } \\
\text { religion }\end{array}$ & Complementarity & Triangulation & $\begin{array}{l}\text { Participants' } \\
\text { definitions of } \\
\text { key variables }\end{array}$ & $\begin{array}{l}\text { Measure key } \\
\text { variables }\end{array}$ & Interpretation & \multicolumn{2}{|c|}{$\begin{array}{l}\text { Survey Instrument with structured } \& \\
\text { open ended questions } \\
\text { Thematic analysis } \\
\text { Descriptive statistics } \\
\text { Inferential - chi square, t- test, } \\
\text { ANOVA }\end{array}$} \\
\hline $\begin{array}{l}\text { Hodge \& Limb } \\
\text { (2009a) }\end{array}$ & $\begin{array}{l}\text { Validation of an } \\
\text { ecomap } \\
\text { assessment } \\
\text { tool for use with } \\
\text { American Indians }\end{array}$ & Triangulation & Triangulation & $\begin{array}{l}\text { Strengths and } \\
\text { limitations of } \\
\text { the concept } \\
\text { and } \\
\text { suggestions } \\
\text { for } \\
\text { improvement }\end{array}$ & $\begin{array}{l}\text { Assess } \\
\text { consistency of } \\
\text { assessment } \\
\text { tool }\end{array}$ & Interpretation & \multicolumn{2}{|c|}{$\begin{array}{l}\text { Questionnaire with structured \& open } \\
\text { ended questions } \\
\text { Constant Comparative Method } \\
\text { Descriptive and Inferential statistics } \\
\text { - Pearson's } r, t \text {-test, ANOVA }\end{array}$} \\
\hline $\begin{array}{l}\text { Hodge \& Limb } \\
\text { (2009b) }\end{array}$ & $\begin{array}{l}\text { Validation of a } \\
\text { spiritual } \\
\text { assessment } \\
\text { tool for use with } \\
\text { American Indians }\end{array}$ & Triangulation & Triangulation & $\begin{array}{l}\text { Strengths and } \\
\text { limitations of } \\
\text { the concept } \\
\text { and } \\
\text { suggestions } \\
\text { for } \\
\text { improvement }\end{array}$ & $\begin{array}{l}\text { Assess } \\
\text { consistency of } \\
\text { assessment } \\
\text { tool }\end{array}$ & Interpretation & \multicolumn{2}{|c|}{$\begin{array}{l}\text { Questionnaire with structured \& open } \\
\text { ended questions } \\
\text { Constant Comparative Method } \\
\text { Descriptive statistics and Inferential } \\
\text { Pearson's } r\end{array}$} \\
\hline $\begin{array}{l}\text { Hodge \& Roby } \\
\text { (2010) }\end{array}$ & $\begin{array}{l}\text { Coping among } \\
\text { women living with } \\
\text { HIV/AIDS }\end{array}$ & Triangulation & Triangulation & $\begin{array}{l}\text { Participants' } \\
\text { coping } \\
\text { strategies }\end{array}$ & $\begin{array}{l}\text { Measure } \\
\text { perceptions } \\
\text { on the } \\
\text { usefulness of } \\
\text { coping } \\
\text { strategies } \\
\text {-Examine } \\
\text { variable } \\
\text { relationships }\end{array}$ & $\begin{array}{l}\text { Data analysis } \\
\text { Interpretation }\end{array}$ & \multicolumn{2}{|c|}{$\begin{array}{l}\text { Questionnaire with structured \& open } \\
\text { ended questions } \\
\text { Constant Comparative Method } \\
\text { Descriptive statistics } \\
\text { Inferential - chi-square, Pearson's } r, t \text { - } \\
\text { test, ANOVA }\end{array}$} \\
\hline $\begin{array}{l}\text { Huyck, } \\
\text { Ayalon, \& } \\
\text { Yoda (2007) }\end{array}$ & $\begin{array}{l}\text { Validation of } \\
\text { assessment tool }\end{array}$ & Triangulation & Triangulation & $\begin{array}{l}\text { Participants' } \\
\text { experiences }\end{array}$ & $\begin{array}{l}\text { Assess } \\
\text { changes in } \\
\text { outcome over } \\
\text { time }\end{array}$ & $\begin{array}{l}\text { Design level } \\
\text { Interpretation }\end{array}$ & $\begin{array}{l}\text { In-depth } \\
\text { interviews } \\
\text { Observations } \\
\text { Grounded } \\
\text { theory }\end{array}$ & \begin{tabular}{|l|} 
Survey \\
\\
Descriptive and \\
Inferential statistics \\
-Cronbach's Alpha
\end{tabular} \\
\hline
\end{tabular}




\begin{tabular}{|c|c|c|c|c|c|c|c|c|}
\hline $\begin{array}{l}\text { Lee, H. \& } \\
\text { Eaton (2009) }\end{array}$ & $\begin{array}{l}\text { Older adult } \\
\text { Korean } \\
\text { immigrants' } \\
\text { perceptions \& } \\
\text { response to } \\
\text { financial abuse }\end{array}$ & Triangulation & Triangulation & $\begin{array}{l}\text { Perceptions } \\
\text { and responses } \\
\text { to a financial } \\
\text { abuse case } \\
\text { vignette }\end{array}$ & $\begin{array}{l}\text { Measure key } \\
\text { variables }\end{array}$ & Interpretation & $\begin{array}{l}\text { In-depth } \\
\text { interviews } \\
\text { Grounded } \\
\text { theory }\end{array}$ & $\begin{array}{l}\text { Survey } \\
\text { Descriptive and } \\
\text { inferential statistics } \\
\text {-Logistic Regression }\end{array}$ \\
\hline $\begin{array}{l}\text { Lee, S. et al. } \\
(2008)\end{array}$ & $\begin{array}{l}\text { HIV vaccine } \\
\text { acceptability } \\
\text { among ethnically } \\
\text { diverse persons }\end{array}$ & Complementarity & Triangulation & $\begin{array}{l}\text { Social issues, } \\
\text { concerns, } \\
\text { barriers and } \\
\text { motivators of } \\
\text { HIV vaccine } \\
\text { acceptability }\end{array}$ & $\begin{array}{l}\text { Measure } \\
\text { consumer } \\
\text { preferences }\end{array}$ & Interpretation & $\begin{array}{l}\text { Focus Group } \\
\text { Thematic } \\
\text { analysis }\end{array}$ & $\begin{array}{l}\text { Conjoint analysis } \\
\\
\text { Descriptive and } \\
\text { inferential statistics } \\
\text { - } t \text {-test } \\
\text {-ANOVA }\end{array}$ \\
\hline $\begin{array}{l}\text { Leslie, } \\
\text { Weckerly, } \\
\text { Plemmons, } \\
\text { Landsverk, \& } \\
\text { Eastman } \\
(2004)\end{array}$ & $\begin{array}{l}\text { Evaluation of a } \\
\text { project protocol }\end{array}$ & Triangulation & Triangulation & $\begin{array}{l}\text { Experiences } \\
\text { of } \\
\text { stakeholders }\end{array}$ & $\begin{array}{l}\text { Measure key } \\
\text { variables }\end{array}$ & Interpretation & $\begin{array}{l}\text { Interviews } \\
\text { Thematic } \\
\text { analyses }\end{array}$ & $\begin{array}{l}\text { Survey } \\
\text { Descriptive and } \\
\text { inferential statistics } \\
\text {-T-test } \\
\text {-Chi-Square }\end{array}$ \\
\hline Maiter (2004) & $\begin{array}{l}\text { Cultural sensitivity } \\
\text { and cultural } \\
\text { competence in } \\
\text { child protection }\end{array}$ & Triangulation & Triangulation & $\begin{array}{l}\text { Perceptions of } \\
\text { parents and } \\
\text { child welfare } \\
\text { workers }\end{array}$ & $\begin{array}{l}\text { Examine } \\
\text { differences } \\
\text { in responses } \\
\text { between } \\
\text { parents and } \\
\text { child welfare } \\
\text { professionals }\end{array}$ & Interpretation & \multicolumn{2}{|c|}{$\begin{array}{l}\text { Survey- Instrument with structured \& } \\
\text { open ended questions } \\
\text { Content analysis } \\
\text { Descriptive statistics } \\
\text { Inferential - Mann-Whitney U }\end{array}$} \\
\hline $\begin{array}{l}\text { McAuley, } \\
\text { McCurry, } \\
\text { Knapp, } \\
\text { Beecham, \& } \\
\text { Sleed (2006) }\end{array}$ & $\begin{array}{l}\text { Evaluation of a } \\
\text { family support } \\
\text { program }\end{array}$ & Triangulation & Triangulation & $\begin{array}{l}\text {-Participants' } \\
\text { experiences } \\
\text { with parenting } \\
\text { stress } \\
\text {-Perceptions } \\
\text { of services }\end{array}$ & $\begin{array}{l}\text { Assess } \\
\text { maternal and } \\
\text { child } \\
\text { wellbeing }\end{array}$ & $\begin{array}{l}\text { Design level } \\
\text { Interpretation }\end{array}$ & $\begin{array}{l}\text { In-depth } \\
\text { Interviews } \\
\text { No specified } \\
\text { method of } \\
\text { data analysis }\end{array}$ & $\begin{array}{l}\text { Survey } \\
\text { Descriptive and } \\
\text { Inferential Statistics } \\
\text { - ANOVA }\end{array}$ \\
\hline $\begin{array}{l}\text { McCarter } \\
(2009)\end{array}$ & $\begin{array}{l}\text { Minority } \\
\text { overrepresentation } \\
\text { in the juvenile } \\
\text { justice system }\end{array}$ & Triangulation & Triangulation & $\begin{array}{l}\text { Participants' } \\
\text { perceptions }\end{array}$ & $\begin{array}{l}\text { Examine } \\
\text { variable } \\
\text { relationships }\end{array}$ & Interpretation & $\begin{array}{l}\text { In-depth } \\
\text { Interviews } \\
\text { Coding }\end{array}$ & $\begin{array}{l}\text { Secondary Data } \\
\text { Analysis } \\
\text { Descriptive and } \\
\text { Inferential Statistics } \\
\quad \text { - Logistic } \\
\text { regression }\end{array}$ \\
\hline
\end{tabular}




\begin{tabular}{|c|c|c|c|c|c|c|c|c|}
\hline $\begin{array}{l}\text { Nicotera } \\
(2008)\end{array}$ & $\begin{array}{l}\text { Measurement of } \\
\text { the concept of } \\
\text { neighborhood }\end{array}$ & Triangulation & Triangulation & $\begin{array}{l}\text { Children's } \\
\text { descriptions of } \\
\text { their } \\
\text { neighborhood } \\
\text { s and } \\
\text { neighboring } \\
\text { experiences }\end{array}$ & $\begin{array}{l}\text { Measure } \\
\text { neighborhood } \\
\text { characteristics }\end{array}$ & $\begin{array}{l}\text { Data Analysis } \\
\text { Interpretation }\end{array}$ & $\begin{array}{l}\text { Document } \\
\text { Analysis } \\
\text { Content } \\
\text { Analysis } \\
\text { Constant } \\
\text { Comparative }\end{array}$ & $\begin{array}{l}\text { Secondary Data } \\
\text { Transformed } \\
\text { qualitative data } \\
\\
\text { Descriptive } \\
\text { Statistics } \\
\text { Inferential Statistics } \\
\text { Chi- Square }\end{array}$ \\
\hline $\begin{array}{l}\text { Park, Knapp, } \\
\text { Shin, \& } \\
\text { Kinslow (2009) }\end{array}$ & $\begin{array}{l}\text { Social engagement } \\
\text { experiences of } \\
\text { older men in } \\
\text { assisted living } \\
\text { facilities }\end{array}$ & $\begin{array}{l}\text { Purpose not } \\
\text { Stated }\end{array}$ & Explanatory & $\begin{array}{l}\text { Participants' } \\
\text { social } \\
\text { engagement } \\
\text { experiences }\end{array}$ & $\begin{array}{l}\text { Measure well- } \\
\text { being \& } \\
\text { social } \\
\text { engagement } \\
\text { variables }\end{array}$ & $\begin{array}{l}\text { Data } \\
\text { collection }\end{array}$ & $\begin{array}{l}\text { In-depth } \\
\text { interviews } \\
\text { Coding }\end{array}$ & $\begin{array}{l}\text { Survey } \\
\text { Descriptive } \\
\text { Statistics } \\
\text { Inferential Statistics } \\
\text { Chi- Square } \\
\text { T-tests } \\
\end{array}$ \\
\hline $\begin{array}{l}\text { Rakfeldt } \\
(2005)\end{array}$ & $\begin{array}{l}\text { Evaluation of } \\
\text { dialectical } \\
\text { behavior therapy }\end{array}$ & $\begin{array}{l}\text { Purpose not } \\
\text { Stated }\end{array}$ & Embedded & $\begin{array}{l}\text { Participants' } \\
\text { experiences } \\
\text { and } \\
\text { interactions }\end{array}$ & $\begin{array}{l}\text { Measure } \\
\text { outcomes }\end{array}$ & Design level & $\begin{array}{l}\text { In-depth } \\
\text { Interviews } \\
\text { Focus Groups }\end{array}$ & $\begin{array}{l}\text { Survey } \\
\text { Descriptive } \\
\text { Statistics } \\
\text { Inferential Statistics } \\
\text { Chi- Square } \\
\text { T-tests }\end{array}$ \\
\hline Redman (2008) & $\begin{array}{l}\text { Coping-related } \\
\text { motives for } \\
\text { substance use }\end{array}$ & Triangulation & Triangulation & $\begin{array}{l}\text { Respondents' } \\
\text { meanings \& } \\
\text { motives }\end{array}$ & $\begin{array}{l}\text { Examine } \\
\text { variable } \\
\text { relationships }\end{array}$ & Data analysis & \multicolumn{2}{|c|}{$\begin{array}{l}\text { Survey with open-ended and closed } \\
\text { questions } \\
\text { - Grounded theory for qualitative data } \\
\text { - Descriptive and Inferential Statistics } \\
\text { - Discriminant Analysis }\end{array}$} \\
\hline $\begin{array}{l}\text { Redmond, } \\
\text { Guerin, \& } \\
\text { Devitt (2008) }\end{array}$ & $\begin{array}{l}\text { Attitudes of social } \\
\text { work students }\end{array}$ & Expansion & Triangulation & $\begin{array}{l}\text { Participants' } \\
\text { future plans }\end{array}$ & $\begin{array}{l}\text { Measure } \\
\text { variables }\end{array}$ & Interpretation & $\begin{array}{l}\text { Survey with o } \\
\text { questions } \\
\text { Focus group } \mathrm{f} \\
\text { collection } \\
\text { - Content Ana } \\
\text { - Descriptive } \\
\text { - Friedman }\end{array}$ & $\begin{array}{l}\text { 1-ended and closed } \\
\text { qualitative data } \\
\text { is for qualitative data } \\
\text { Inferential Statistics } \\
\text { Analysis of Variance }\end{array}$ \\
\hline
\end{tabular}




\begin{tabular}{|c|c|c|c|c|c|c|c|c|}
\hline $\begin{array}{l}\text { Sanders, R., \& } \\
\text { Roach (2007) }\end{array}$ & $\begin{array}{l}\text { Evaluation of } \\
\text { family support } \\
\text { services }\end{array}$ & Triangulation & Embedded & $\begin{array}{l}\text { Family views } \\
\text { and service } \\
\text { expectations }\end{array}$ & $\begin{array}{l}\text { Measure } \\
\text { outcomes and } \\
\text { change after } \\
\text { intervention }\end{array}$ & $\begin{array}{l}\text { - Design level } \\
\text { - } \\
\text { Interpretation }\end{array}$ & $\begin{array}{l}\text { In-depth } \\
\text { interviews } \\
\text { - No specified } \\
\text { method of } \\
\text { qualitative } \\
\text { data analysis } \\
\end{array}$ & $\begin{array}{l}\text { Survey } \\
\text { Descriptive and } \\
\text { Inferential statistics } \\
\text { - } T \text {-test } \\
\end{array}$ \\
\hline $\begin{array}{l}\text { Sanders, S., } \\
\text { Ott, Kelber, \& } \\
\text { Noonan (2008) }\end{array}$ & Grief reactions & Triangulation & Triangulation & $\begin{array}{l}\text { Lived } \\
\text { experiences }\end{array}$ & $\begin{array}{l}\text { Measure grief } \\
\text { levels }\end{array}$ & Interpretation & $\begin{array}{l}\text { In-depth } \\
\text { Interviews } \\
\text { Coding }\end{array}$ & $\begin{array}{l}\text { Survey } \\
\text { Descriptive and } \\
\text { Inferential Statistics } \\
\quad \cdot T \text {-tests } \\
\text { Chi-square }\end{array}$ \\
\hline $\begin{array}{l}\text { Smith \& } \\
\text { Roberts (2009) }\end{array}$ & $\begin{array}{l}\text { Young parents' } \\
\text { antenatal \& } \\
\text { postnatal needs }\end{array}$ & Triangulation & Triangulation & $\begin{array}{l}\text { Add detail } \\
\text { and context to } \\
\text { survey } \\
\text { findings }\end{array}$ & $\begin{array}{l}\text { Measure } \\
\text { variables }\end{array}$ & Interpretation & $\begin{array}{l}\text { Focus Group } \\
\text { Thematic } \\
\text { analysis }\end{array}$ & $\begin{array}{l}\text { Survey } \\
\text { Descriptive and } \\
\text { Inferential Statistics } \\
\text { - Chi-square } \\
\text { (Fishers' Exact Test) }\end{array}$ \\
\hline $\begin{array}{l}\text { Tolmie et al. } \\
\text { (2009) }\end{array}$ & $\begin{array}{l}\text { Needs of older } \\
\text { people in cardiac } \\
\text { rehabilitation }\end{array}$ & Triangulation & Triangulation & $\begin{array}{l}\text { Participants' } \\
\text { experiences }\end{array}$ & $\begin{array}{l}\text { Measure key } \\
\text { study } \\
\text { variables }\end{array}$ & Interpretation & $\begin{array}{l}\text { In-depth } \\
\text { interviews } \\
\text { Framework } \\
\text { Analysis }\end{array}$ & $\begin{array}{l}\text { Surveys } \\
\\
\text { Descriptive and } \\
\text { Inferential Statistics } \\
\text { - Kruskal-Wallis } \\
\text { - One Way } \\
\text { ANOVA } \\
\text { - Mann-Whitney U }\end{array}$ \\
\hline $\begin{array}{l}\text { Varas-Díaz \& } \\
\text { Marzán- } \\
\text { Rodríguez } \\
(2007)\end{array}$ & $\begin{array}{l}\text { Role of Emotions } \\
\text { in HIV/AIDS } \\
\text { Stigmatization }\end{array}$ & Development & Exploratory & $\begin{array}{l}\text { Perceptions of } \\
\text { people living } \\
\text { with } \\
\text { HIV/AIDS } \\
\text { and associated } \\
\text { emotions } \\
\end{array}$ & $\begin{array}{l}\text {-Measure the } \\
\text { different } \\
\text { emotions } \\
\text {-Examine } \\
\text { differences }\end{array}$ & $\begin{array}{l}\text { Data } \\
\text { collection }\end{array}$ & $\begin{array}{l}\text { In-depth } \\
\text { Interviews } \\
\text { Thematic } \\
\text { analysis }\end{array}$ & $\begin{array}{l}\text { Survey } \\
\text { Descriptive and } \\
\text { Inferential Statistics } \\
\quad \cdot t \text {-tests }\end{array}$ \\
\hline $\begin{array}{l}\text { Waldrop } \\
\text { (2007) }\end{array}$ & Caregiver grief & Triangulation & Embedded & $\begin{array}{l}\text { Participants' } \\
\text { experiences }\end{array}$ & $\begin{array}{l}\text { Measure } \\
\text { dimensions of } \\
\text { distress }\end{array}$ & Design level & \multicolumn{2}{|c|}{$\begin{array}{l}\text { Survey with open-ended and closed } \\
\text { questions } \\
\text { - Open and axial coding of qualitative } \\
\text { data } \\
\text { - Descriptive and Inferential Statistics } \\
\text { - } t \text {-tests }\end{array}$} \\
\hline
\end{tabular}




\begin{tabular}{|c|c|c|c|c|c|c|c|c|}
\hline $\begin{array}{l}\text { Yamatani, } \\
\text { Engel, \& } \\
\text { Spjeldnes } \\
(2009)\end{array}$ & $\begin{array}{l}\text { Caseload } \\
\text { standards for child } \\
\text { welfare workers }\end{array}$ & Triangulation & Triangulation & $\begin{array}{l}\text { Examine } \\
\text { caseload } \\
\text { service tasks } \\
\text { in their } \\
\text { context }\end{array}$ & $\begin{array}{l}\text { Classify \& } \\
\text { record case } \\
\text { management } \\
\text { tasks }\end{array}$ & Interpretation & $\begin{array}{l}\text { Focus Group } \\
\text { No specified } \\
\text { method of } \\
\text { data analysis) }\end{array}$ & $\begin{array}{l}\text { Direct Observation } \\
\text { Document Review } \\
\text { Descriptive statistics }\end{array}$ \\
\hline Yoo (2003) & $\begin{array}{l}\text { Organizational } \\
\text { characteristics } \\
\text { and client } \\
\text { outcomes }\end{array}$ & Expansion & Triangulation & $\begin{array}{l}\text { Perceptions of } \\
\text { the } \\
\text { organization }\end{array}$ & $\begin{array}{l}\text { Measure key } \\
\text { outcomes e.g. } \\
\text { job } \\
\text { satisfaction }\end{array}$ & Interpretation & $\begin{array}{l}\text { In-depth } \\
\text { interviews } \\
\text { Grounded } \\
\text { theory }\end{array}$ & $\begin{array}{l}\text { Document Review } \\
\text { Survey } \\
\\
\text { Descriptive } \\
\text { Statistics and } \\
\text { Inferential } \\
\text { - T-test } \\
\end{array}$ \\
\hline Yoon (2009) & $\begin{array}{l}\text { Role of leadership, } \\
\text { community } \\
\text { cohesion and } \\
\text { mental health in } \\
\text { community } \\
\text { rebuilding after a } \\
\text { flood }\end{array}$ & Complementarity & Embedded & $\begin{array}{l}\text { - Views on } \\
\text { elected } \\
\text { leadership } \\
\text { - Identify } \\
\text { community } \\
\text { assets } \\
\text { - Check } \\
\text { convergent } \\
\text { validity of } \\
\text { data from } \\
\text { surveys }\end{array}$ & $\begin{array}{l}\text { Measure } \\
\text { outcomes and } \\
\text { examine } \\
\text { variable } \\
\text { relationships }\end{array}$ & Design level & $\begin{array}{l}\text {-In-depth } \\
\text { Interviews } \\
\text {-Document } \\
\text { review } \\
\text { No specified } \\
\text { method of } \\
\text { qualitative } \\
\text { data analysis }\end{array}$ & $\begin{array}{l}\text { Survey } \\
\text { Descriptive and } \\
\text { Inferential Statistics } \\
\text { - Spearman's } \\
\text { correlation }\end{array}$ \\
\hline
\end{tabular}




\section{Common Methods of Qualitative and Quantitative Data Collection}

Table 3 presents all the methods of qualitative and quantitative data collection used in the reviewed articles. In-depth interviews were the most common qualitative data collection method, accounting for $41 \%$ of all the qualitative techniques reported in the articles, whereas surveys represented $55 \%$ of all the quantitative data collection techniques.

Table 3. Methods of Qualitative and Quantitative Data Collection

\begin{tabular}{|c|c|c|c|}
\hline \multicolumn{2}{|c|}{ Qualitative } & \multicolumn{2}{|c|}{$\underline{\text { Quantitative }}$} \\
\hline Type & Count (\%) & Type & Count (\%) \\
\hline In-depth Interviews & $21(41)$ & Surveys & $30(55)$ \\
\hline Focus Groups & $13(25)$ & $\begin{array}{l}\text { Close-ended Questions in } \\
\text { Questionnaire }\end{array}$ & $12(22)$ \\
\hline \multirow[t]{2}{*}{$\begin{array}{l}\text { Open-ended Questions in } \\
\text { Questionnaire }\end{array}$} & $12(24)$ & $\begin{array}{l}\text { Observations with Rating } \\
\text { Scale }\end{array}$ & $4(7)$ \\
\hline & & Secondary Data & $3(5)$ \\
\hline Document Review & $3(6)$ & Document Review & $2(4)$ \\
\hline \multirow[t]{2}{*}{ Web-based Survey } & $2(4)$ & Transformed Data & $2(4)$ \\
\hline & & Conjoint Analysis & $1(2)$ \\
\hline Total & 51 & & 54 \\
\hline
\end{tabular}

\section{Common Methods of Quantitative and Qualitative Data Analysis}

Table 4 shows all the qualitative and quantitative data analysis methods employed in the reviewed articles. Thematic analysis accounted for $29 \%$ of all the qualitative data analysis techniques and $t$-test represented $30 \%$ of all the quantitative data analysis methods. Whereas all the quantitative data analysis techniques were elaborated, $12 \%$ of the qualitative data analysis techniques were not specified.

\section{Key Areas Addressed by the Qualitative and Quantitative Strands}

The key areas addressed by the qualitative and quantitative strands in the reviewed articles are summarized in Table 5. Drawing from Table 5 three elements that mixed methods research adds to social work were categorized. First, mixed methods research adds voices of study participants to social work research. Understanding participants' experiences and views constituted $68 \%$ of the stated foci of the qualitative strand in the mixed method studies. The need to understand and incorporate client views and perspectives is central to social work practice and research because it is the means through which social workers are equipped to become the voice for their respective clientele (Fernandez, 2008). 
Table 4. Methods of Qualitative and Quantitative Data Analysis

\begin{tabular}{lrlr}
\hline \multirow{2}{*}{ Type } & Qualitative & & Quantitative \\
Thematic Analysis & Count (\%) & Type & Count (\%) \\
Open Coding & $14(29)$ & t-test & $18(30)$ \\
Content Analysis & $10(21)$ & Chi-Square & $12(19)$ \\
Constant Comparative Method & $6(12)$ & ANOVA & $11(20)$ \\
Not specified & $6(12)$ & Pearson's $r$ & $7(12)$ \\
Grounded Theory & $6(12)$ & Cronbach's alpha & $3(5)$ \\
Framework Analysis & $5(10)$ & Logistic Regression & $2(3)$ \\
& $1(2)$ & Mann-Whitney U & $2(3)$ \\
& & Discriminant Analysis & $1(2)$ \\
& & Friedman's Analysis of Variance & $1(2)$ \\
& & Spearman's Correlation & $1(2)$ \\
Total & & Structural Equation Modeling & $1(2)$ \\
\hline
\end{tabular}

Table 5. Focus of Qualitative and Quantitative Parts

\begin{tabular}{lrlr}
\hline Focus of Qualitative Part & Number (\%) & Focus of Quantitative Part & Number (\%) \\
\hline Participants' Experiences & $20(39)$ & $\begin{array}{l}\text { Measure/Describe key } \\
\text { constructs } \\
\text { Examine variable relationships }\end{array}$ & $78(69)$ \\
$\begin{array}{l}\text { Participants' } \\
\begin{array}{l}\text { Views/Perspectives e.g. with } \\
\text { program or intervention }\end{array}\end{array}$ & $15(29)$ & Examine differences \\
$\begin{array}{l}\text { Examine Process e.g. } \\
\text { caseload service tasks in } \\
\text { their naturalistic setting, } \\
\text { interactions between case } \\
\text { workers and clients }\end{array}$ & $7(16)$ & Exs & $7(13)$ \\
$\begin{array}{l}\text { Validity Issues e.g. check } \\
\text { convergent validity }\end{array}$ & $7(14)$ & Validate Instrument Structure & $3(5)$ \\
$\begin{array}{l}\text { Instrument Development } \\
\text { Total }\end{array}$ & $1(2)$ & & \\
\hline
\end{tabular}


Second, mixed methods research allows for comprehensive analyses of phenomena. Central to this category is the realization that a single phenomenon can be best understood from different angles, such as perceptions held by the participants, underlying factors or process as well as the measurable or quantifiable trends and outcomes. To this end the foci of the reviewed studies illustrated these aspects with $29 \%$ of the focus areas related to capturing participants' views/perspectives, 16\% examining processes, $69 \%$ measuring or describing key constructs, and $13 \%$ examining variable relationships. For instance, in his study of the factors associated with community rebuilding after a flood, Yoon (2009) used quantitative methods to examine relationships between various outcomes, whereas qualitative methods were used to gather views about elected leadership from diverse informants and to understand other important community assets. After data analyses, Yoon found that while elected leaders' ability to mobilize resources was significantly related to financial recovery, this specific community's symbolic meaning as the first town chartered by African Americans in the United States was also an important asset in the rebuilding process. Thus, the ability to simultaneously measure outcomes and capture the context, processes, and participants' views enable mixed methods research to achieve a more holistic analysis of phenomena.

Third, mixed methods research enhances the validity of findings. A basic foundation of mixed methods research is the notion of triangulation whereby "two or more methods that have offsetting biases are... intentionally used to assess the same conceptual phenomenon" (Greene et al., 1989, p. 256). In addition, "triangulation of methods can provide opportunities for testing alternative interpretations," such as the influence of context on the observed results (Polit \& Beck, 2007, p. 310). Validation of instrument structure accounted for $5 \%$ of all the quantitative strand foci whereas efforts to enhance validity, such as by checking for convergent validity, represented $14 \%$ of the qualitative strand foci. For example, in their study of well-being of mothers with families under stress, McAuley and colleagues (2006) used quantitative methods to assess the levels of parenting stress and depression among the mothers and qualitative methods to gather their experiences with parenting stress. After analyzing the quantitative data, these authors found that the mothers had high levels of parenting stress and depression, results which were supported by the symptoms reported by the mothers in the qualitative interviews. Validity is enhanced when comparisons of results obtained across the quantitative and qualitative methods and data support each other (Greene et al., 1989).

\section{Discussion and Social Work Implications}

To build our knowledge on the current state of mixed methods research in social work, this literature review examined the common quantitative and qualitative methods used by social work researchers and inferred the value of mixed methods research for social work based on the foci of the qualitative and quantitative strands. Similar to reviews in other disciplines, surveys and interviews were the most common methods of quantitative and qualitative data collection, respectively (Bryman, 2006; O'Cathain et al., 2007). The dominance of in-depth interviews is acceptable because in-depth interviews facilitate access into participants' cultures and perspectives, and shift authority away from the researcher to the participants (Goodman, 2001). The recognition of document 
review as a method of gathering both qualitative and quantitative data is a positive development that may allow increased use of existing agency data by social work researchers, and thereby promote collaboration with social work practitioners.

Thematic analysis and $t$-tests were the most common methods of qualitative and quantitative data analysis, respectively. Of particular concern 6 out of the $52(12 \%)$ qualitative data analysis techniques used in the reviewed articles were not specified. This finding raises questions regarding the incorporation of qualitative research procedures. Failure to elaborate on the qualitative data analysis methods may indicate possible difficulties in ensuring an acceptable balance to the integration of the quantitative and qualitative techniques. Cognizant of the challenges associated with achieving competency in both qualitative and quantitative research, the promotion of team work when conducting mixed methods studies is encouraged (Padgett, 2009; Plano Clark, Huddleston-Casas, Churchill, O’Neil Green, \& Garrett, 2008). In addition, explicit teaching of mixed methods research in social work is warranted to familiarize social workers with mixed methods research terminology and procedures.

The studies reviewed demonstrate that there are added advantages in using mixed methods research in social work. By allowing comprehensive analyses of phenomena mixed methods research echoes principles of social work practice that require social workers to "study things holistically, in context, and from more than one frame of reference" (Cowger \& Menon, 2001, p. 477). Given that social workers represent vulnerable populations, capturing the voices of our client groups may be integral to their emancipation and empowerment (Fernandez, 2008; O'Cathain et al., 2007). For these reasons, the teaching and use of mixed methods research remain integral to social work.

This article would not be complete without an acknowledgment of the limitations of this study. First, the reviewed articles do not represent a comprehensive list of all possible mixed methods articles in social work. Second, the researcher's subjective judgment influenced the review process and understanding of the reviewed articles. Despite these limitations, this study represents a preliminary attempt to systematically review the use and value of mixed methods research in published social work articles. Most important, it provides social work researchers with examples of published mixed methods studies and advances our understanding of the value of mixed methods research in social work.

\section{Conclusion}

This literature review examined the common quantitative and qualitative methods in published social work articles and what mixed methods research may add to social work. Mixed methods research's ability to simultaneously capture measurable outcomes, context, participants' voices, and process underscores its value to social work research. Giving voice to study participants and allowing for a holistic analysis of complex social problems may advance the development of useful knowledge and provide a richer understanding of the populations that we serve. Even though the idea of mixing qualitative and quantitative methods is not new to social work practice, there is need for more research to understand how mixed methods research is taught and embraced in 
social work. Social work educators and researchers may play a pivotal role in ensuring adequate training to conduct, consume, and assess mixed methods research.

\section{References}

Abel, E., \& Campbell, M. (2009). Student-centered learning in an advanced social work practice course: Outcomes of a mixed methods investigation. Social Work Education, 28(1), 3-17. doi:10.1080/02615470701844423

Antle, B., \& Collins, W. (2009). The impact of a spirituality-based support group on selfefficacy and well-being of African American breast cancer survivors: A mixed methods design. Social Work \& Christianity, 36(3), 286-300.

Axinn, W. G., \& Pearce, L. D. (2006). Mixed method data collection strategies. Cambridge, UK: Cambridge University Press.

Ayón, C., \& Lee, C. D. (2009). Building strong communities: An evaluation of a Neighborhood Leadership Program in a diverse urban area. Journal of Community Psychology, 37(8), 975-986. doi:10.1002/jcop.20343

Beecher, B. (2009a). The medical model, mental health practitioners, and individuals with schizophrenia and their families. Journal of Social Work Practice, 23(1), 9-20.

Beecher, B. (2009b). Mental health practitioners' views of the families of individuals with schizophrenia and barriers to collaboration: A mixed methods study. Journal of Family Social Work, 12(3), 264-282.

Bellamy, C. D., Garvin, C., MacFarlane, P., Mowbray, O. P., Mowbray, C. T., \& Holter, M. C. (2006). An analysis of groups in consumer-centered programs. American Journal of Psychiatric Rehabilitation, 9(3), 219-240. doi:10.1080/15487760600962152

Berger, L. K., Otto-Salaj, L. L., Stoffel, V. C., Hernandez-Meier, J., \& Gromoske, A. N. (2009). Barriers and facilitators of transferring research to practice: An exploratory case study of motivational interviewing. Journal of Social Work Practice in the Addictions, 9(2), 145-162. doi:10.1080/15332560902806199

Boateng, A. (2009). A mixed methods analysis of social capital of Liberian refugee women in Ghana. Journal of Sociology and Social Welfare, 36(3), 59-81.

Bryan, V., Flaherty, C., \& Saunders, C. (2010). Supporting adoptive families: Participant perceptions of a statewide peer mentoring and support program. Journal of Public Child Welfare, 4(1), 91-112. doi:10.1080/15548730903563178

Bryman, A. (2006). Integrating quantitative and qualitative research: How is it done? Qualitative Research, 6(1), 97-113.

Butler, S. S. (2006). Evaluating the Senior Companion Program: A mixed-method approach. Journal of Gerontological Social Work, 47(1/2), 45-70. 
Campbell, J. (2008). Stakeholders' views of legal and advice services for people admitted to psychiatric hospital. Journal of Social Welfare \& Family Law, 30(3), 219-232. doi:10.1080/09649060802550659

Carpenter, J., Barnes, D., Dickinson, C., \& Wooff, D. (2006). Outcomes of interprofessional education for community mental health services in England: The longitudinal evaluation of a postgraduate program. Journal of Interprofessional Care, 20(2), 145-161.

Chan, E., Chi, S., Ching, S., \& Lam, S. (2010). Interprofessional education: The interface of nursing and social work. Journal of Clinical Nursing, 19(1-2), 168-176. doi:10.1111/j.1365-2702.2009.02854.x

Chan, E., Mok, E., Po-ying, A., \& Man-chun, J. (2009). The use of interdisciplinary seminars for the development of caring dispositions in nursing and social work students. Journal of Advanced Nursing, 65(12), 2658-2667. doi:10.1111/j.13652648.2009.05121.x

Cheung, M. (2008). Resilience of older immigrant couples: Long-term marital satisfaction as a protective factor. Journal of Couple \& Relationship Therapy, 7(1), 19-38.

Connelly, L. M. (2009). Mixed methods studies. MEDSURG Nursing, 18(1), 31-32.

Cowger, C. D., \& Menon, G. (2001). Integrating qualitative and quantitative research methods. In B. A. Thyer (Ed.), The handbook of social work research methods (pp. 473-484). Thousand Oaks, CA: Sage Publications.

Creswell, J. W. (2008). Educational research: Planning, conducting, and evaluating quantitative and qualitative research ( $3^{\text {rd }}$ ed.). Upper Saddle River, NJ: Pearson/ Prentice Hall.

Creswell, J. W., \& Plano Clark, V. L. (2007). Designing and conducting mixed methods research. Thousand Oaks, CA: Sage Publications.

Creswell, J. W., \& Plano Clark, V. L. (2011). Designing and conducting mixed methods research $\left(2^{\text {nd }}\right.$ ed.). Thousand Oaks, CA: Sage Publications.

Daftary, D. (2010). Democratic decentralization from the bottom up: The comparative effect of wealth and electoral capital on elected leaders' distribution of development. Social Development Issues, 32(2), 42-54.

Desimone, L. M. (2009). Complementary methods for policy research. In G. Sykes, B. Schneider, \& D. N. Plank (Eds.), Handbook of educational policy research (pp. 163175). New York, NY: Routledge.

Fernandez, E. (2008). Unraveling emotional, behavioral and educational outcomes in a longitudinal study of children in foster-care. The British Journal of Social Work, 38(7), 1283-1301.

Flick, U. (2009). An introduction to qualitative research. Los Angeles, CA: Sage Publications. 
Freedman, D. (2009). Local food environments: They're all stocked differently. American Journal of Community Psychology, 44(3/4), 382-393. doi:10.1007/s10464009-9272-6

Gallagher, P. A., Malone, D., \& Ladner, J. R. (2009). Social-psychological support personnel: Attitudes and perceptions of teamwork supporting children with disabilities. Journal of Social Work in Disability \& Rehabilitation, 8(1), 1-20. doi:10.1080/15367100802665540

Gioia, D. (2006). Examining work delay in young adults with schizophrenia. American Journal of Psychiatric Rehabilitation, 9(3), 167-190. doi:10.1080/15487760600961543

Gioia, D., \& Brekke, J. S. (2003). Knowledge and use of workplace accommodations and protections by young adults with schizophrenia: A mixed method study. Psychiatric Rehabilitation Journal, 27(1), 59-68. doi:10.2975/27.2003.59.68

Goodman, H. (2001). In-depth interviews. In B. A. Thyer (Ed.), The handbook of social work research methods (pp. 309-319). Thousand Oaks, CA: Sage Publications.

Greene, J. C. (2007). Mixed methods in social inquiry ( $1^{\text {st }}$ ed.). San Francisco, CA: Jossey-Bass.

Greene, J. C., Caracelli, V. J., \& Graham, W. F. (1989). Toward a conceptual framework for mixed-method evaluation designs. Educational Evaluation and Policy Analysis, 11(3), 255-274.

Grinnell, Jr., R. M., \& Unrau, Y. A. (2008). Social work research and evaluation: Foundations of evidence-based practice ( $8^{\text {th }}$ ed.) New York, NY: Oxford University Press.

Hernandez, B., McDonald, K., Lepera, N., Shahna, M., Wang, T., \& Levy, J. M. (2009). Moving beyond misperceptions: The provision of workplace accommodations. Journal of Social Work in Disability \& Rehabilitation, 8(3/4), 189-204. doi:10.1080/15367100903202755

Hodge, D., \& Boddie, S. (2007). Social workers' personal spiritual characteristics and their conceptualizations of spirituality and religion: A mixed method study. Journal of Religion \& Spirituality in Social Work, 26(1), 53-70.

Hodge, D., \& Limb, G. (2009a). Establishing the preliminary validity of Spiritual EcoMaps with Native Americans. Clinical Social Work Journal, 37(4), 320-331. doi:10.1007/s10615-009-0203-7

Hodge, D. R., \& Limb, G. E. (2009b). Spiritual histories and Native Americans: A mixed method validation study. Journal of Social Service Research, 35(4), 285-296. doi:10.1080/01488370903110795

Hodge, D., \& Roby, J. (2010). Sub-Saharan African women living with HIV/AIDS: An exploration of general and spiritual coping strategies. Social Work, 55(1), 27-37. doi:10.1093/sw/55.1.27 
Hopson, L. M., \& Steiker, L. K. H. (2008). Methodology for evaluating an adaptation of evidence-based drug abuse prevention in alternative schools. Children \& Schools, 30(2), 160.

Huyck, M., Ayalon, L., \& Yoder, J. (2007). Using mixed methods to evaluate the use of a caregiver strain measure to assess outcomes of a caregiver support program for caregivers of older adults. International Journal of Geriatric Psychiatry, 22(2), 160165. doi:10.1002/gps.1707

Johnson, B., \& Christensen, L. (2007). Educational research: Quantitative, qualitative, and mixed approaches ( $3^{\text {rd }}$ ed.). Thousand Oaks, CA: Sage Publications.

Johnson, B., \& Turner, L. A. (2003). Data collection strategies in mixed methods research. In A. Tashakkori \& C. Teddlie (Eds.), Handbook of mixed methods in social and behavioral research (pp. 297-319). Thousand Oaks, CA: Sage Publications.

Klenke, K. (2008). Qualitative research in the study of leadership. Bingley, UK: Emerald Group Publishing Limited.

Lee, H., \& Eaton, C. K. (2009). Financial abuse in elderly Korean immigrants: Mixed analysis of the role of culture on perception and help-seeking intention. Journal of Gerontological Social Work, 52(5), 463-488. doi:10.1080/01634370902983138

Lee, S., Brooks, R. A., Newman, P. A., Seiden, D., Sangthong, R., \& Naihua, D. (2008). HIV vaccine acceptability among immigrant Thai residents in Los Angeles: A mixedmethod approach. AIDS Care, 20(10), 1161-1168. doi:10.1080/09540120701855375

Leslie, L. K., Weckerly, J., Plemmons, D., Landsverk, J., \& Eastman, S. (2004). Implementing the American Academy of Pediatrics Attention-Deficit/Hyperactivity Disorder Diagnostic guidelines in primary care settings. Pediatrics, 114(1), 129-140.

Lodico, M. G., Spaulding, D. T., \& Voegtle, K. H. (2006). Methods in educational research: From theory to practice. San Francisco, CA: John Wiley and Sons.

Maiter, S. (2004). Considering context and culture in child protection services to ethnically diverse families: An example from research with parents from the Indian Subcontinent (South Asians). Journal of Social Work Research \& Evaluation, 5(1), 63-80

McAuley, C., McCurry, N., Knapp, M., Beecham, J., \& Sleed, M. (2006). Young families under stress: Assessing maternal and child well-being using a mixed-methods approach. Child \& Family Social Work, 11(1), 43-54.

McCarter, S. A. (2009). Legal and extralegal factors affecting minority overrepresentation in Virginia's juvenile justice system: A mixed-method study. Child and Adolescent Social Work Journal, 26(6), 533 - 544. doi: 10.1007/s10560009-0185-x 
Moher, D., Liberati, A., Tetzlaff, J., Altman, D. G., \& The PRISMA Group. (2009). Preferred Reporting Items for Systematic Reviews and Meta-Analyses: The PRISMA Statement. PLOS Medicine, 6(7), e1000097. doi:10.1371/journal.pmed.1000097

Nicotera, N. (2008). Children speak about neighborhoods: using mixed methods to measure the construct neighborhood. Journal of Community Psychology, 36(3), 333351. doi:10.1002/jcop. 20197

O'Cathain, A., Murphy, E., \& Nicholl, J. (2007). Why, and how, mixed methods research is undertaken in health services research in England: A mixed methods study. BioMed Central Health Services Research, 7(85). doi:10.1186/1472-6963-7-85

Padgett, D. K. (1998). Qualitative methods in social work research: Challenges and rewards. Thousand Oaks, CA: Sage Publications.

Padgett, D. K. (2008). Qualitative methods in social work research ( $2^{\text {nd }}$ ed.). Thousand Oaks, CA: Sage Publications.

Padgett, D. K. (2009). Qualitative and mixed methods in social work knowledge development. Social Work, 54(2), 101-105.

Park, N., Knapp, M. A., Shin, H., \& Kinslow, K. M. (2009). Mixed methods study of social engagement in assisted living communities: Challenges and implications for serving older men. Journal of Gerontological Social Work, 52(8), 767-783. doi:10.1080/01634370903285541

Patton, M. Q. (2002). Qualitative research and evaluation methods ( $3^{\text {rd }}$ ed.). Thousand Oaks, CA: Sage Publications.

Plano Clark, V. L., Huddleston-Casas, C.A., Churchill, S. L., O’Neil Green, D., \& Garrett, A. L. (2008). Mixed methods approaches in family science research. Journal of Family Issues OnlineFirst. doi: 10.1177/0192513X08318251

Polit, D. E., \& Beck, C. T. (2006). Essentials of nursing research: Methods, appraisal, and utilization $\left(6^{\text {th }}\right.$ ed). Philadelphia, PA: Lippincott Williams \& Wilkins

Rakfeldt, J. (2005). Dialectical Behavior Therapy with transitional youth: Preliminary findings. Best Practice in Mental Health, 1(2), 61-76.

Redman, D. (2008). Coping-related substance use motives and stressful life experiences among people with a history of incarceration. Journal of Social Work Practice in the Addictions, 8(4), 490-510.

Redmond, B., Guerin, S., \& Devitt, C. (2008). Attitudes, perceptions and concerns of student social workers: First two years of a longitudinal study. Social Work Education, 27(8), 868-882. doi:10.1080/02615470701844233

Rubin, A., \& Babbie, E. R. (2008). Research methods for social work (6 $6^{\text {th }}$ ed.). Belmont, CA: Thompson \& Brooks/Cole. 
Sanders, R., \& Roach, G. (2007). Closing the gap? The effectiveness of referred access family support services. Child \& Family Social Work, 12(2), 161-171. doi:10.1111/j.1365-2206.2006.00455.x

Sanders, S., Ott, C., Kelber, S., \& Noonan, P. (2008). The experience of high levels of grief in caregivers of persons with Alzheimer's disease and related dementia. Death Studies, 32(6), 495-523.

Smith, D., \& Roberts, R. (2009). Young parents' perceptions of barriers to antenatal and postnatal care. British Journal of Midwifery, 17(10), 620, 622-627.

Tashakkori, A., \& Creswell, J. W. (2007). The new era of mixed methods. Journal of Mixed Methods Research, 1(1), 3-7.

Teddlie, C., \& Yu, F. (2007). Mixed methods sampling: A typology with examples. Journal of Mixed Methods Research, 1(1), 77-100.

Tolmie, E., Lindsay, G., Kelly, T., Tolson, D., Baxter, S., \& Belcher, P. (2009). Are older patients' cardiac rehabilitation needs being met? Journal of Clinical Nursing, 18(13), 1878-1888. doi:10.1111/j.1365-2702.2009.02798.x

Varas-Díaz, N., \& Marzán-Rodríguez, M. (2007). The emotional aspect of AIDS stigma among health professionals in Puerto Rico. AIDS Care, 19(10), 1247-1257.

Waldrop, D. P. (2007). Caregiver grief in terminal illness and bereavement: A mixedmethods study. Health \& Social Work, 32(3), 197-206.

Weathington, B. L., Cunningham, C. J. L., \& Pittenger, D. J. (2010). Research methods for the behavioral and social sciences. Hoboken, NJ: John Wiley \& Sons.

Yamatani, H., Engel, R., \& Spjeldnes, S. (2009). Child welfare worker caseload: What's just right? Social Work, 54(4), 361-368.

Yegidis, B. L., \& Weinbach, R. W. (2008). Research methods for social workers (6 ${ }^{\text {th }}$ ed.). Boston, MA: Pearson/Allyn and Bacon.

Yoo, J. (2002). The relationship between organizational variables and client outcomes: A case study in child welfare. Administration in Social Work, 26(2), 39-61.

Yoon, I. (2009). A mixed-method study of Princeville's rebuilding from the flood of 1999: Lessons on the importance of invisible community assets. Social Work, 54(1), $19-28$.

\section{Author note:}

Address correspondence to: Josphine Chaumba, Ph.D., Department of Social Work, The University of North Carolina at Pembroke, One University Drive, P. O. Box 1510, Pembroke, NC 28372. Email: josphine.chaumba@uncp.edu 